\title{
Possible relationship between Helicobacter pylori infection and cap polyposis of the colon
}

Taiji Akamatsu, M.D., Ph.D. ${ }^{1)}$, Naoshi Nakamura, M.D., Ph.D. ${ }^{2)}$, Yoko Kawamura, M.D. ${ }^{2)}$, Akihiro Shinji, M.D. ${ }^{2)}$, Nobuyuki Tateiwa, M.D. ${ }^{1)}$, Yasuhide Ochi, M.D., Ph.D. ${ }^{2)}$, Tsutomu Katsuyama, M.D., Ph.D. ${ }^{3)}$, and Kendo Kiyosawa, M.D., Ph.D. ${ }^{2)}$

1) Department of Endoscopy, Shinshu University Hospital, Shinshu University School of Medicine, Matsumoto, Japan

2) Internal Medicine, Gastroenterology, Shinshu University School of Medicine, Matsumoto, Japan

3) Department of Laboratory Medicine, Shinshu University School of Medicine, Matsumoto, Japan

Correspondence: Taiji Akamatsu, M.D., Ph.D., Department of Endoscopy, Shinshu University Hospital, 3-1-1 Asahi, Matsumoto, Nagano 390-8621, Japan TEL+81-263-37-2804 FAX:+81-263-32-9412 E-mail: ta07260@hsp.md.shinshu-u.ac.jp

Running Title: Helicobacter pylori infection and cap polyposis

Key words: cap polyposis, inflammatory bowel disease, treatment, Helicobacter pylori, eradication therapy 


\begin{abstract}
Background: Cap polyposis is a rarely encountered disease that describes multiple distinctive inflammatory colonic polyps located from the rectum to the distal colon. The etiology of this disease is still unknown, and no specific treatment has been established. Aim: We report 3 cases of cap polyposis that were cured following eradication therapy for Helicobacter pylori infection. Methods and Results: Three women were referred to Shinshu University Hospital because of mucoid and/or bloody diarrhea. Laboratory data showed hypoproteinemia in all cases; markers of inflammation such as C-reactive protein were negative. Colonoscopy revealed multiple sessile polyps attached with mucus on the apices of the mucosal folds in the rectum and/or the sigmoid colon. The intervening mucosa was normal. Microscopic examinations of biopsy specimens taken from sessile polyps revealed inflamed mucosa with elongated tortuous crypts attenuated towards the mucosal surface. A granulation tissue "cap" was observed on the surface of the mucosa. Various treatments were unsuccessful including administration of metronidazole or prednisolone, avoidance of straining at defecation, and surgical or endoscopic resection. All were diagnosed with $H$. pylori infection in the stomach. H. pylori was not detected in the biopsy specimens from the colonic inflammatory polyps by immunohistochemical study using polyclonal anti-H. pylori antibody. After successful eradication therapy the clinical symptoms improved. Disappearance of cap polyposis was confirmed by colonoscopy in all 3 cases.
\end{abstract} Conclusion: We speculate that $H$. pylori infection might play a role in the pathogenesis of cap polyposis. 


\section{Introduction}

Cap polyposis is an inflammatory bowel disease first reported in 1985 by Williams et al. ${ }^{1}$ in abstract form and concerned the histopathological findings of 15 cases. Consequently, this disease was not generally known until 2 clinical cases were described in 1993 by Campbell et al. ${ }^{2)}$. To date, 18 cases have been reported in MEDLINE. This rare disease has characteristic clinical, endoscopic, and pathological features, and is therefore relatively easy to diagnose if a clinician or/and a pathologist is aware if it. The etiology of the disease is still unknown, and no specific or effective treatment has been established. Consequently, patients with cap polyposis generally suffer from this disease for a long time.

Recently, Oiya et al. ${ }^{3)}$ reported a patient with cap polyposis who was cured by Helicobacter pylori eradication therapy prompting us to attempt this treatment for 3 cases of cap polyposis of long duration. All patients were remarkably improved.

\section{Case Reports}

Case 1: A 33-year-old woman was seen in 1993 for treatment of proctitis. She had suffered from mucous bloody stool for 2 years and been diagnosed with mucosal prolapse syndrome of the rectum. Past and family medical histories were unremarkable. Physical examination was normal except for pretibial pitting edema. Laboratory data showed hypoproteinemia (total protein 4.8 $\mathrm{g} / \mathrm{dl}$, albumin $2.8 \mathrm{~g} / \mathrm{dl}$ ). Markers of inflammation such as C-reactive protein (CRP) were normal. Colonoscopy showed multiple sessile polyps on the apices of the mucosal folds in the rectum (Fig. 1a). The surface of these lesions was reddish and adhered by mucus. Multiple white specks were recognized in the area surrounding the polyps. The intervening mucosa was normal, and no diverticular disease was present. Microscopic findings of biopsy specimens revealed inflamed mucosa with elongated tortuous crypts attenuated towards the mucosal surface, and a granulation tissue "cap" on the surface of mucosa (Fig. 1b). Culture of feces and serum anti-amoebic antibody were negative. On the basis of these findings, the patient was diagnosed with cap polyposis. Treatment with oral intake of $750 \mathrm{mg}$ /day of metronidazole, or $40 \mathrm{mg} / \mathrm{day}$ of prednisolone and $3.0 \mathrm{~g}$ /day of salazosulphapyrisine were not effective. Home alimentation with digestive element and a suppository of a small dose of steroids $(0.5-1.0 \mathrm{mg} / \mathrm{day}$ of betamethazone) were given along with the recommendation to avoid straining at defecation. Her symptoms and endoscopic findings showed no remarkable change. Endoscopic treatment with endoscopic mucosal resection and endoscopic cauterization using argon plasma coagulation 
was attempted on in August 2000 resulting in improvement of symptoms and an increase in serum total protein (to total protein $6.0 \mathrm{mg} / \mathrm{dl}$ ); however, symptoms recurred 3 months after endoscopic treatment and colonoscopy showed multiple sessile polyps attached with mucus in the rectum.

H. pylori infection was diagnosed by culture and histology of gastric biopsy specimens. Immunohistochemical study using polyclonal anti-H. pylori antibody (DAKO Corp., Carpenteria, CA) showed no $H$. pylori in biopsy specimens obtained from inflammatory sessile polyps in the rectum (Fig. 1c). After written informed consent, she received eradication therapy with $40 \mathrm{mg}$ of rabeprazole, $1500 \mathrm{mg}$ of amoxicillin, and $800 \mathrm{mg}$ of clarithromycin daily for 7 days. This was begun in October 2002. Eradication therapy was successful, and her symptoms such as mucous bloody stool and pretibial edema were also immediately improved. After 3 months, colonoscopy revealed disappearance of multiple sessile polyps (Fig. 1d). Serum total protein normalized to 7.0 $\mathrm{g} / \mathrm{dl}$, and the patient has had no recurrence for 18 months since eradication therapy of H. pylori.

Case 2: A 50-year-old woman was referred to our hospital in September 1996 complaining of mucous bloody stool, tenesmus, and $8 \mathrm{~kg}$ weight loss. She had been suffering from mucous bloody stool for 10 months and had been diagnosed with ulcerative colitis. She had been treated with oral salazosulfapyridine and prednisolone, but her symptoms had become gradually more severe. Past and family medical histories were unremarkable. Physical examination was normal. Laboratory data showed almost normal except hypoproteinemia (total protein $5.2 \mathrm{~g} / \mathrm{dl}$, albumin $3.3 \mathrm{~g} / \mathrm{dl}$ ); CRP was not increased. Colonoscopy revealed multiple sessile polyps on the apices of the mucosal folds in the rectum and the sigmoid colon. The surface of these lesions was reddish with adherent mucus, and multiple white specks in the area surrounding them (Fig. 2a). The intervening mucosa was normal, and no diverticular disease was observed. Microscopic findings of biopsy specimens showed inflamed mucosa with elongated tortuous crypts attenuated towards the mucosal surface. Culture of feces and serum anti-amoebic antibody were negative. Alphaa-1-antitrypsin clearance test showed positive $(428.9 \mathrm{~mL} /$ day $)$. The progressive hypoproteinemia was thought to be due to protein losing enteropathy. On the basis of these findings, the patient was diagnosed with cap polyposis. Treatment with oral intake of $750 \mathrm{mg} / \mathrm{day}$ of metronidazole for 2 weeks was not effective. Avoidance of straining at defecation did not improve her symptoms. Total parenteral nutrition and enemas of $40 \mathrm{mg} / \mathrm{day}$ of prednisolone improved her symptoms and colonoscopic findings temporarily, but frequent mucous diarrhea recurred immediately after the resumption of eating. Surgical resection of the sigmoid colon was 
performed in November 1997 due to resistance to the conservative therapies. Unfortunately, her symptoms such as mucous bloody stool and tenesmus recurred 6 months after resection. Colonoscopy showed multiple sessile polyps in the anal side of anastomotic portion, and multiple flat-topped protruding-type lesions in the oral site of anastomotic lesions (Fig. 2b). Microscopic findings of biopsy specimens revealed cap polyposis (Fig. 2c).

She was diagnosed with $H$. pylori infection by rapid urease test and histology of gastric biopsy specimens in another hospital during a health screening in January 2002. H. pylori was positive in both examinations, and she received eradication therapy with $60 \mathrm{mg}$ of lansoprazole, $1500 \mathrm{mg}$ of amoxicillin, and $800 \mathrm{mg}$ of clarithromycin all taken daily for 7 days. Eradication therapy was successful, and her symptoms remarkably improved. Serum total protein normalized (Total protein $6.9 \mathrm{~g} / \mathrm{dl}$ ), and colonoscopy revealed the disappearance of multiple flat-topped protruding-type lesions (Fig. 2d) 6 months after eradication therapy for H. pylori. The patient has remained free of disease for 26 months following eradication therapy of $H$. pylori. Notably, $H$. pylori was not detected in biopsy specimens that were taken from inflammatory polyps of the colon before eradication therapy by immunohistochemical study using anti-H. pylori antibody.

Case 3: A 53-year-old woman was referred to our hospital in June 2002 for mucous bloody stool, watery diarrhea, and pretibial edema for 17 years. She had been diagnosed with ulcerative colitis in the referring hospital. She had been treated with oral intake of $3.0 \mathrm{~g} / \mathrm{day}$ of salazosulphapyrisine or $750 \mathrm{mg} /$ day of metronidazole without improvement. Treatment with oral prednisolone (30 mg/day) had been temporarily effective, but her symptoms had recurred upon reducing the dosage. Past and family medical histories were unremarkable. Physical examination was normal. Laboratory data showed hypoproteinemia (total protein $5.2 \mathrm{~g} / \mathrm{dl}$, albumin $3.4 \mathrm{~g} / \mathrm{dl}$ ), but other data, including CRP, were normal. Colonoscopy showed variform protruding lesions that were separated by normal mucosa in the rectum and the sigmoid colon (Fig. 3a). No diverticular disease was seen. Microscopic findings of biopsy specimens revealed inflamed mucosa with elongated tortuous crypts. Culture of feces and serum anti-amoebic antibody were negative. On the basis of these findings, the patient was diagnosed with cap polyposis. Treatment with topical administration of steroid hormone (corresponding to $20 \mathrm{mg}$ of prednisolone daily) enemas reduced her symptoms, and her serum protein gradually increased (total protein $7.7 \mathrm{~g} / \mathrm{dl}$ ). However, she was later diagnosed with glaucoma and diabetes mellitus and steroids were discontinued. $H$. pylori infection was identified by gastric mucosal culture and histology. Immunohistochemical study using anti-H. pylori antibody failed to show $H$. pylori in biopsy 
specimens obtained from the variform protruding mucosa in the rectum and the sigmoid colon. After written informed consent, she received eradication therapy with $60 \mathrm{mg}$ of lansoprazole, $1500 \mathrm{mg}$ of amoxicillin, and $800 \mathrm{mg}$ of clarithromycin taken daily for 7 days Eradication therapy was successful, and her symptoms were immediately improved. Colonoscopy at 3 months after eradication therapy revealed the disappearance of variform protruding mucosa (Fig. 3b). She has remained well with no recurrence up to 15 months after eradication therapy.

\section{Discussion}

We have encountered 6 cases of cap polyposis including these 3 cases since 1983. The other 3 cases were reported previously as "unclassified colitis"4)-6). We reviewed the 18 reported clinical cases diagnosed with cap polyposis ${ }^{2) 3) 7-17)}$ and our 6 cases. The median age of the patients was 48.5 years (range, 12-76 years) showing that cap polyposis can occur over a wide range of age. The sex ratio favored women (men 6: women 18). The most frequent complaints were mucoid and/or bloody diarrhea. Laboratory data usually revealed hypoproteinemia due to protein-losing enteropathy. Shiomi et al. ${ }^{18)}$ reported a case of cap polyposis (included in reference 8) in which it was demonstrated that the extent of cap polyposis recognized on colonoscopy was congruous with excessive protein loss from the lesion, as shown on scintigraphy with human serum albumin labeled with Tc-99m DTPA. In two of our cases including a previously reported case $^{6}$, , protein-losing enteropathy was confirmed by al-antitripsin clearance test, and hypoproteinemia improved in association with resolution of cap polyposis. The affected region of cap polyposis was generally recognized in the rectum and the sigmoid colon; however, protuberant lesions were observed from the rectum to the right side colon in 2 cases $^{3) 15)}$, and from the rectum to the descending colon in 4 cases $^{2) 8) 9111)}$.

The characteristic endoscopic findings are multiple sessile polyps attached with mucus on the apices of the mucosal folds, and the intervening mucosa was normal. Esaki et al. ${ }^{13)}$ analyzed endoscopic findings in 4 cases, and reported that endoscopic features were of two types: a semipedunculated type and a flat-topped protruding type. In our previously reported case ${ }^{5)}$, the first colonoscopy showed multiple flushed edematous mucosa, and these findings developed into typical multiple sessile polyps after 4 months. Gehenot et al. ${ }^{7)}$ and Kajihara et al. ${ }^{10)}$ reported the same development in endoscopic findings. Based on these findings, multiple flushed edematous mucosa separated by normal mucosa are thought to be initial and/or mild endoscopic findings of cap polyposis. 
Various treatment procedures have been attempted. 5-aminosalicylic acid agent was administered in 9 of the 24 cases, but no remarkable improvement was recognized in any case. Steroid hormone was administered in 10 of 24 cases again without remarkable improvement in 7 cases; temporal improvement was recognized in the remaining 3 cases. Metronidazole was administered in 12 cases, and clinical symptoms and endoscopic findings subsequently disappeared in $4(33.3 \%)^{4) 5(10) 16)}$, with temporary improvement in clinical symptoms in the other 2 cases $^{7) 13)}$. However, no remarkable improvement was recognized in the remaining 6 cases. Surgical resection of the affected colon was performed in 9 of 24 cases, but recurrence of symptoms and endoscopic findings within 12 months following the operation was recognized in 5 of these 9 cases $(55.6 \%)$. Thus while surgical resection may be temporally effective, but recurrences are common. Endoscopic mucosal resection with cauterization using high-frequency current was administered in case 1. Temporal improvement of symptoms was recognized, but recurrence of symptoms and endoscopic findings was observed after 6 months. This treatment has the same risk of recurrence as surgical resection of cap polyposis. Of interest, 4 cases of spontaneous resolution of cap polyposis have been reported ${ }^{6) 13(15) 17)}$.

Williams et al. ${ }^{1)}$ and Campbell et al. ${ }^{2)}$ suggested that chronic mechanical stimulation by abnormal rectosigmoid motility might be important in the pathogenesis of cap polyposis, based on its histological resemblance to mucosal prolapse syndrome. Oriuchi et al. ${ }^{11)}$ reported 2 cases of successful treatment of cap polyposis by avoidance of intraluminal trauma; one was cured by avoidance of straining at defecation, and another was resolved after double-barreled transverse colostomy. This report was thought to support the hypothesis of Williams et al. and Campbell et al. We recommended avoidance of straining at defecation in 3 of our cases including a previously reported case ${ }^{6}$, but this practice resulted in no remarkable improvement in any case. Total parenteral nutrition, which is expected to reduce mechanical stimulation of the digesta for the rectosigmoid mucosa, was performed in 2 of our cases including a previously reported case ${ }^{6)}$, and symptoms and endoscopic findings improved temporally, but recurrence was immediately after resumption of eating. Based on these clinical courses, we consider that chronic mechanical stimulation might be a worsening factor for cap polyposis, and that the resemblance of histological findings of this disease to those of mucosal prolapse syndrome, such as the existing of elastin ${ }^{2}$, might be represent a secondary change in the development of cap polyposis.

On the basis of these our 3 cases and the case reported by Oiya et al. ${ }^{3)}$, there appears to be a relationship cap polyposis and $H$. pylori infection. However, no $H$. pylori were detected in the 
colonic mucosa obtained from multiple inflammatory polyps in any cases. It is possible that other as yet unknown bacteria sensitive to the antibiotic combination used to treat $H$. pylori may actually be the cause. For example, Shimizu et al. ${ }^{16)}$ reported a case of cap polyposis with verotoxin-nonproducing Escherichia coli O18 infection. In that case, levofloxacin, a broad-spectrum quinolone antibiotic, successfully eradicated the Escherichia coli, but the patient's symptoms and endoscopic findings remained unchanged.

$H$. pylori infection is thought to be related to Menetrier's disease, which is characterized as hypertrophic gastritis with protein-losing gastroenteropathy. Some cases of Menetrier's disease cured by eradication therapy for $H$. pylori have been reported ${ }^{19)-21)}$. A possible role of transforming growth factor $\alpha$ and a relationship between hepatocyte growth factor and elongated gastric mucosa have been reported ${ }^{22) 23)}$. These growth factors are related to cytokine networks, which are influenced by the immune response to inflammation caused by $H$. pylori infection ${ }^{24)}$. Characteristic histological findings of cap polyposis include inflamed mucosa with elongated tortuous crypts and inflammatory granulomatous tissue on the surface of the mucosa. Multiple polypoid lesions are thought to be composed of elongated tortuous crypts, which resemble the histological finding of Menetrier's disease. Therefore, we suggest that cap polyposis might be hypertrophic change of the large intestine influenced by systemic immune stimulation due to persistent $H$. pylori infection.

The effectiveness of metronidazole for cap polyposis is inconsistent. Gehenot et al. ${ }^{7)}$ reported a case of cap polyposis occurring in the postoperative course of pelvic surgery, and supposed that infection by some unknown microorganism was related to the occurrence of cap polyposis because oral intake of metronidazole was temporarily effective to reduce symptoms. While metronidazole is used in the treatment of $H$. pylori infection, it is only effective for reliable eradication when given as part of a multidrug regimen which may partly explain the inconsistency of metronidazole for cap polyposis. Further examination of the role of $H$. pylori infection in cap polyposis will be enhanced by examination of the $H$. pylori status of other cases, by the response to therapy, and by the recurrence of disease coinciding with failure of $H$. pylori eradication therapy. The response to anti-H. pylori therapy in cases of cap polyposis without coincident $H$. pylori infection, if they exist, would also be of interest.

In conclusion, we recommend testing for $H$. pylori in all cases of cap polyposis. A therapeutic trial of anti-H. pylori eradication therapy should probably be attempted even if $H$. pylori infection is not identified. 


\section{References}

1) Williams GT, Bussey HJR, Morson BC. Inflammatory 'cap'polyps of the large intestine. Br J Surg 1985; 72(suppl):S133.

2) Campbell AP, Cobb CA, Chapman RWG, et al. Cap polyposis-an unusual cause of diarrhoea. Gut 1993; 34: 562-4.

3) Oiya H, Okawa K, Aoki T, et al. Cap polyposis cured by Helicobacter pylori eradication therapy. J Gastroenterol 2002, 37: 463-6.

4) Kamijo N, Yamaguchi K, Akamatsu T, et al. A probable case of amebic colitis. ENDOSCOPIC FORUM for digest dis 1986; 2: 136-40 (Japanese with English abstract).

5) Tsugane E, Akamatsu T, Ohwa O, et al. A case report of unclassified colitis accompanied by pregnancy. ENDOSCOPIC FORUM for digest dis 1989; 5: 258-62 (Japanese with English abstract).

6) Kamijo T, Akamatsu T, Sakai H, et al. A case of unclassified colitis with protein losing enteropathy. Gastroenterol Endosc 1993; 35: 2731-6 (Japanese with English abstract).

7) Gehenot M, Colombel J-F, Wolschies E, et al: Cap polyposis occurring in the postoperative course of pelvic surgery. Gut 1994; $35: 1670-2$.

8) Oshitani N, Moriyama Y, Matsumoto T, et al: Protein-losing enteropathy from cap polyposis. Lancet 1995; 346:1567.

9) Peny MO, Noel JC, Haot J, et al: “Cap polyposis":un syndrome rare. Gastroenterol Clin Biol 1998; 22: 349-352 (French with English abstract).

10) Kajihara H, Uno Y, Ying H, et al: Features of cap polyposis by magnifying colonoscopy. Gastrointest Endosc 2000; 52: 775-8.

11) Oriuchi T, Kinouchi Y, Kimura M, et al: Successful treatment of cap polyposis by avoidance of intraluminal trauma: clues to pathogenesis. Am J Gastroenterol 2000; 95: 2095-8.

12) Tamura S, Onishi S, Ohkawauchi K, et al:A case of “cap polyposis"-like lesion associated with ulcerative colitis:Is this a case of cap polyposis? Am J Gastroenterol 2000; 95:3311-2.

13) Esaki M, Matsumoto T, Kobayashi H, et al: Cap polyposis of the colon and rectum: an analysis of endoscopic findings. Endoscopy 2001; 33: 262-6.

14) Isomoto H, Urata M, Nakagoe T, et al. Proximal extension of cap polyposis comfirmed by colonoscopy. Gastrointest Endosc 2001; 54: 388-91.

15) Sadamoto Y, Jimi S, Harada N, et al. Asymotomatic cap polyposis from the sigmoid colon to 
the cecum. Gastrointest Endosc 2001; 54: 654-6.

16) Shimizu K, Koga H, Iida M, et al. Does metronidazole cure cap polyposis by its anti-inflammatory actions instead of by its antibiotic action? Digest Dis Sci 2002; 47: 1465-8.

17) Ohkawara T, Kato M, Nakagawa S, et al. Spontaneous resolution of cap polyposis: case report. Gastrointest Endosc 2003; 57: 599-602.

18) Shiomi S, Moriyama Y, Oshitani N, et al. A case of cap polyposis investigated by scintigraphy with human serum albumin labeled with Tc-99m DTPA. Clin Nucl Med 1998; 23:521-523.

19) Bayerdorffer E, Ritter MM, Halz R, et al. Healing of protein losing hypertrophic gastropathy by eradication of Helicobacter pylori-Is Helicobacter pylori a pathogenic factor in Menetrier's disease? Gut 1994; 35: 701-4.

20) Groisman GM, George J, Berman D, et al. Resolution of protein-losing hypertrophic lymphocytic gastritis with therapeutic eradication of Helicobacter pylori. Am J Gastroenterol 1994; 89: 1548-51.

21) Kaneko T, Akamatsu T, Gotou A, et al. Remission of Menetrier's disease after a prolonged period with therapeutic eradication of Helicobacter pylori. Am J Gastroenterol 1999; 94: 272-3.

22) Dempsy PJ, Goldenring JR, Soroka CJ, et al. Possible role of transforming growth factor $\alpha$ in the pathogenesis of Menetrier's disease. Supportive evidence from humans and transgenic mice. Gastroenterology 1992: 103; 1950-63.

23) Yasunaga Y, Shinomura Y, Kanayama S, et al. Increased production of interleukin $1 \beta$ and hepatocyte growth factor may contribute to foveolar hyperplasia in enlarged fold gastritis. Gut 1996; 39: 787-94.

24) Messa C, Di Leo A, Greco B, et al. Successful eradicating treatment of Helicobacter pylori in patients with chronic gastritis: Gastric levels of cytokines, epidermal growth factor and polyamines before and after therapy. Immunopharmacol Immunotoxicol 1996; 18: 1-13. 


\section{Figure legends}

Figure 1: Pictures of case 1. (1a) Endoscopic picture of cap polyposis before eradicating $H$. pylori. Multiple sessile polyps, which are attached with mucus, are recognized on the apices of the mucosal fold in the rectum. (1b) Microscopic picture of cap polyposis obtained by endoscopic mucosal resection (jumbo biopsy). Inflamed mucosa with elongated tortuous crypts attenuates towards the mucosal surface, and granulation tissue "cap" is observed on the surface of mucosa (H\&E stain; original magnification $\times 80)$. (1c) Immunostaining reveals no $H$. pylori on the mucosal surface of colonic sessile polyps (Immunostaining for $H$. pylori; original magnification $\times 200$ ). (1d) Endoscopic picture of cap polyposis after 3 months since eradicating $H$. pylori. Multiple sessile polyps are completely disappeared, and only scars due to endoscopic mucosal resection are recognized.

Figure 2: Pictures of case 2. (2a) Endoscopic picture of cap polyposis before eradicating $H$. pylori shows multiple white specks (arrow) in the surrounding of sessile polyps. (2b) Endoscopic picture at the time of recurrence of cap polyposis after 6 months since surgical resection of the affected region. Multiple flat-topped protruding-type lesions are recognized in the oral site of anastomotic lesions. (2c) Microscopic picture of cap polyposis obtained by the conventional biopsy forceps. Granulation tissue is recognized on the surface of inflamed mucosa with elongated crypts (H\&E stain; original magnification $\times 40)$. (2d) Endoscopic picture of cap polyposis after 6 months since eradicating $H$. pylori shows disappearance of multiple flat-topped protruding-type lesions.

Figure 3: Picture of case 3. (3a) Endoscopic picture of cap polyposis before eradicating H. pylori reveals variform protruding lesions in the rectum and the sigmoid colon. Surface of these lesions are reddish, and the intervening mucosa is normal. (3b) Endoscopic picture of the same region as 3a after 3 months since eradicating $H$. pylori shows disappearance of variform protruding lesions. 
Taiji Akamatsu

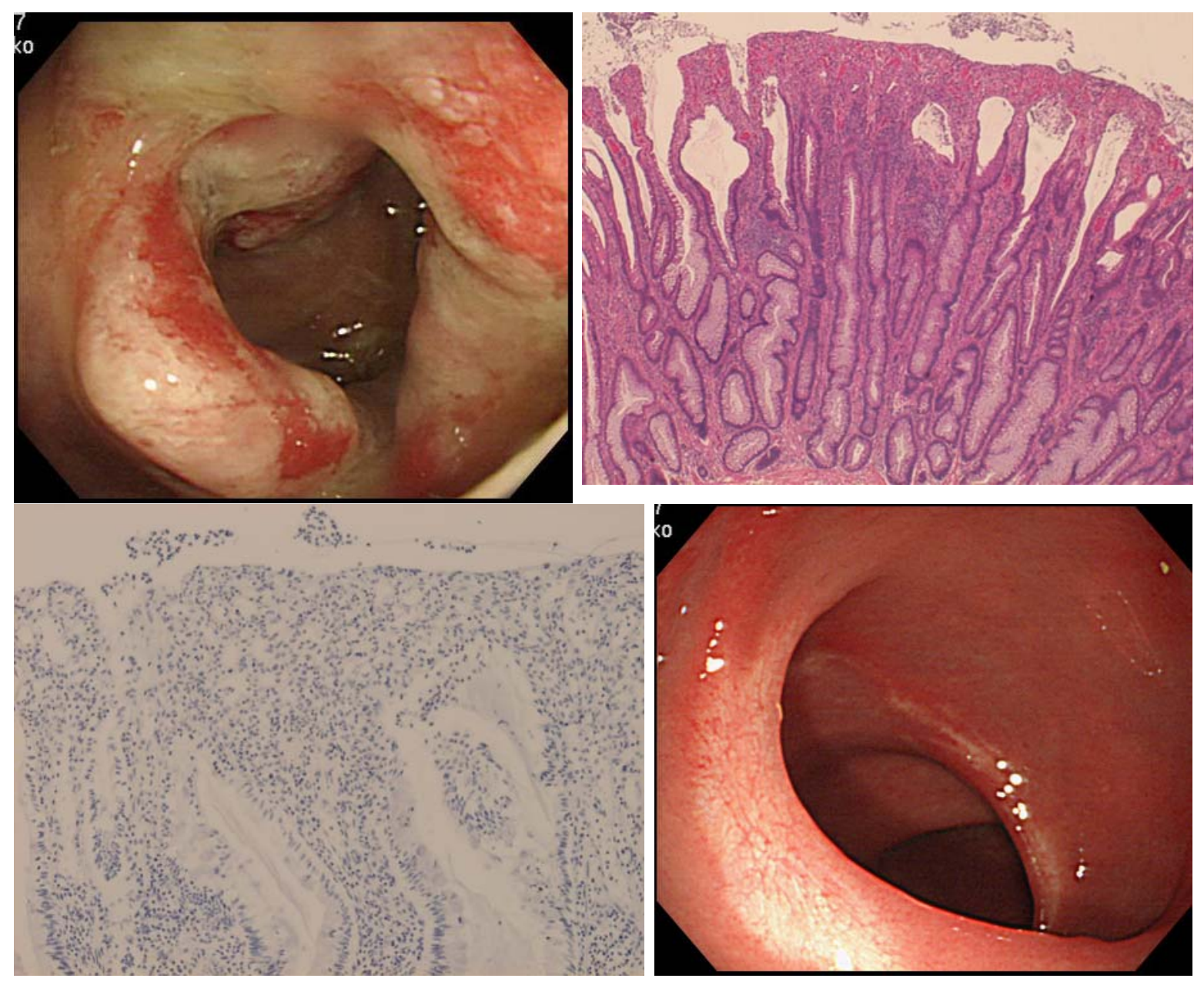


Taiji Akamatsu

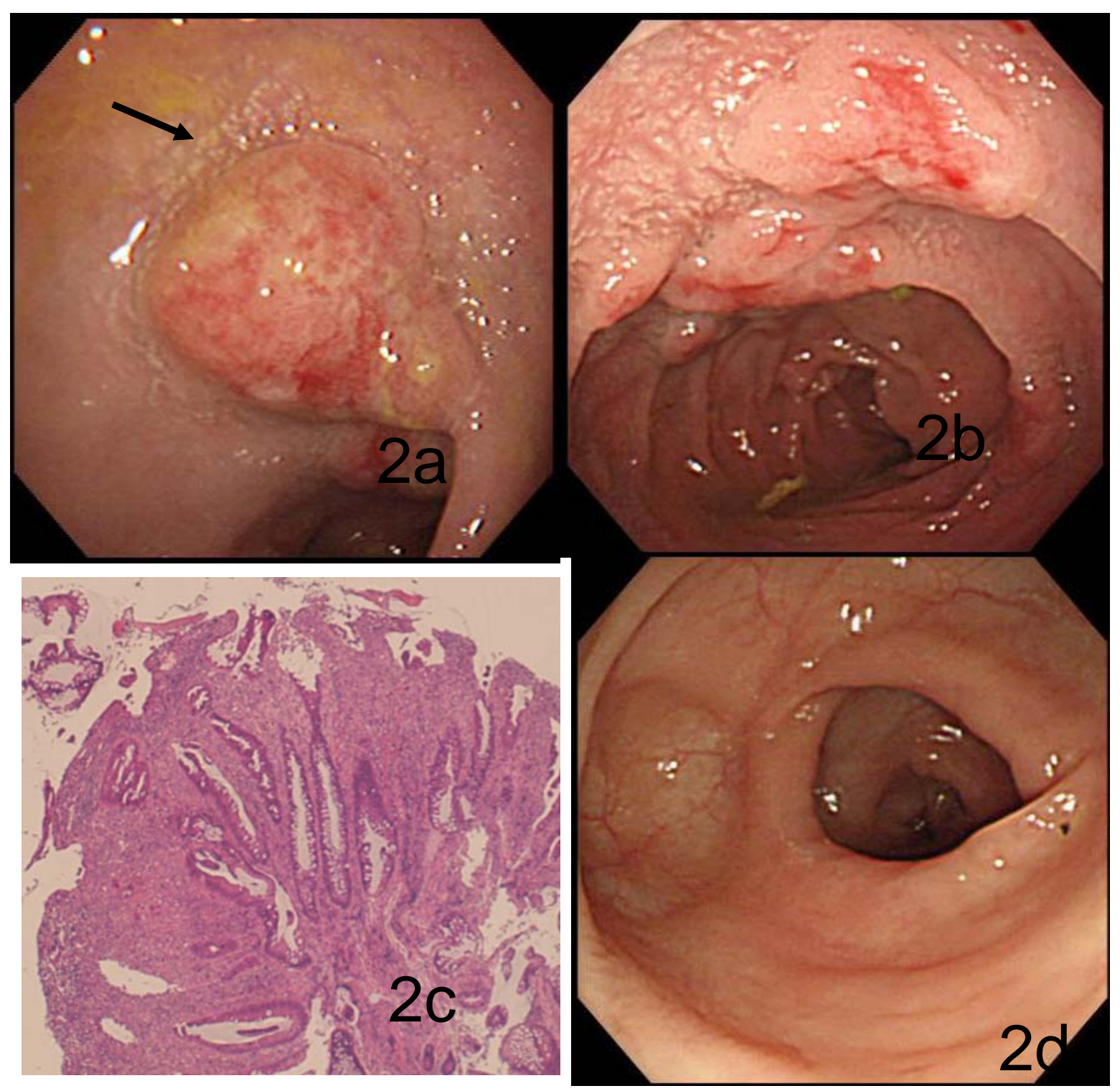


Taiji Akamatsu

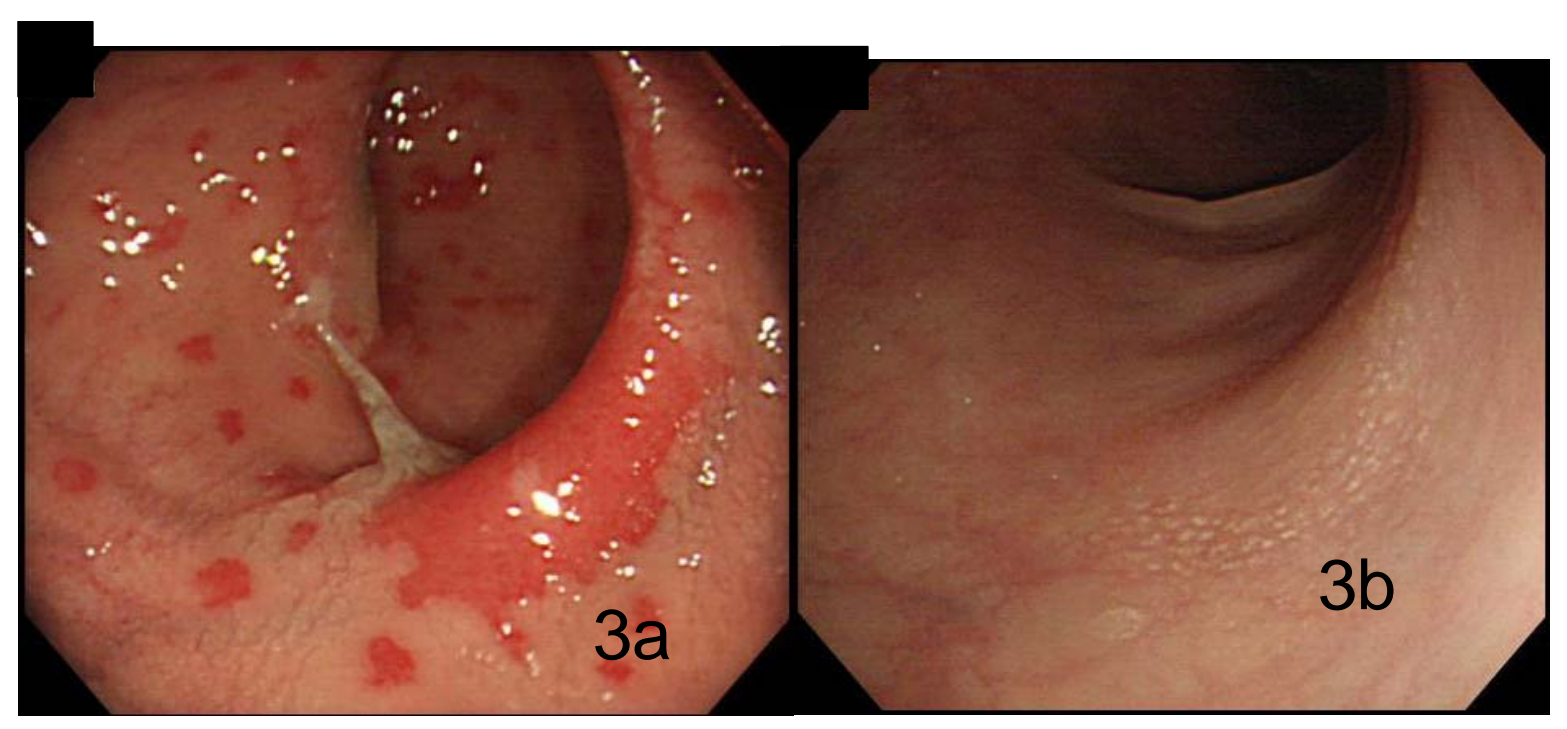

